\title{
Equilibrium Player Choices in Team Contests with Multiple Pairwise Battles*
}

\author{
Hideo Konishi ${ }^{\dagger} \quad$ Chen-Yu Pan Pimitar Simeonov $^{\ddagger}$
}

January 22, 2021

\begin{abstract}
We consider games in which team leaders strategically choose the order of players sent to the battlefield in majoritarian team contests with multiple pairwise battles as in $\mathrm{Fu}, \mathrm{Lu}$, and Pan (2015 American Economic Review). We consider one-shot orderchoice games and battle-by-battle sequential player choice games. We show that as long as the number of players on each team is the same as the number of battles, the equilibrium winning probability of a team and the ex ante expected effort of each player in a multi-battle contest are independent of whether players' assignments are one-shot or battle-by-battle sequential. This equilibrium winning probability and ex ante expected total effort coincide with those where the player matching is chosen totally randomly with an equal probability lottery by the contest organizer. Finally, we show how player choices add subtleties to the equivalence result by examples.
\end{abstract}

\footnotetext{
${ }^{*}$ We thank Stefano Barbieri, Qiang Fu, and Jingfeng Lu for their helpful comments and encouragement. Comments and suggestions from an anonymous referee and associate editor improved the paper tremendously.

${ }^{\dagger}$ Department of Economics, Boston College, USA. hideo.konishi@bc.edu

${ }^{\ddagger}$ Department of International Business, National Chengchi University, Taiwan. panch@nccu.edu.tw

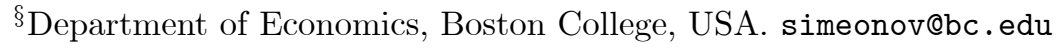




\section{Introduction}

In their influential paper on group contests, Fu, Lu, and Pan (2015) analyze a multi-battle team contest in which players from two rival teams form pairwise matches to compete in distinct component battles: i.e., each player fights exactly one battle in the whole contest. They naturally assume that the winning probability of battles is depicted by a function that is homogeneous of degree zero in players' efforts. ${ }^{1}$ They then show that the outcomes of past battles do not distort the outcomes of future battles, as long as the pairwise matches between the players from the two teams stay the same. That is, (i) the winning probability in each battle (match) is independent of the history of that battle, (ii) the winning probability of a team is independent of the sequence of battles, and (iii) the winning probability of a team is independent of the temporal structure of the component battles (i.e., one-shot or sequential). Moreover, they also show that neither the total expected effort nor the overall outcome of the contest depends on (i) the battle sequence or (ii) the temporal structure. These are quite striking results, which have interesting implications for team competitions in sports and other areas. ${ }^{2}$

In this study, we will consider the team leaders' strategic assignment problem of players to component battles as in the multi-battle team contest of $\mathrm{Fu}, \mathrm{Lu}$, and $\mathrm{Pan}$ (2015). Unlike in $\mathrm{Fu}, \mathrm{Lu}$, and Pan (2015), however, there is no way to fix which players are matched in the next battle in our model, since team leaders can choose who goes next independently. We set up the game in two different ways: a one-shot ordering choice game and a sequential battle-by-battle player choice game.

Equilibrium in a one-shot simultaneous ordering choice game has been analyzed by Hamil-

\footnotetext{
${ }^{1}$ This genre of technology includes many well-accepted models, such as the general Tullock contest and first-price all-pay auction.

${ }^{2}$ Despite of all of these neutrality results by Fu, Lu, and Pan (2015), Barbieri and Serena (2019) show that the expected winners' efforts are higher if battles are held simultaneously than if they are held sequentially under a natural condition.
} 
ton and Romano (1998) under the assumption that each individual match has an exogenously fixed winning probability. Interestingly, they show that there is a mixed strategy equilibrium in which both teams assign the same probability to every ordering of the players, and that the expected winning probability is unique (von Neumann's minimax theorem in a two-person zero-sum game). Although we allow for endogenous effort levels and winning probabilities for each battle, Hamilton and Romano's (1998) analysis is an essential component in obtaining our results.

Although the one-shot ordering choice game is easier to analyze, it may not actually be the most common practice - for example, in the Davis Cup in men's tennis, the team captains announce which players will compete in the next match only after the results of the previous matches are revealed. In the MLB World Series, team managers announce starting pitchers on each game day. Our sequential battle-by-battle player choice game is more suitable for these examples. More importantly, by introducing a sequential player choice for the next battle, we may reveal intriguing insights regarding the following questions: Is it important to have a lead in the early stage (a momentum/discouraging effect)? Should teams reserve strong players for decisive battles in the late stage? Do the results from previous rounds affect leaders' or players' decisions in later stages?

The main result of this paper is that the equilibrium winning probability of a team and the ex ante expected effort of each player in a multi-battle contest are independent of whether players' assignments are one-shot or battle-by-battle sequential as long as the number of players on each team is the same as the number of battles. These equilibrium winning probabilities and ex ante expected total efforts coincide with those where the player matching is chosen totally randomly with an equal probability lottery by the contest organizer.

To prepare for a comprehensive analysis of player choice games in multi-battle team contests, we first reproduce the result by Hamilton and Romano (1998) on one-shot ordering choice games when pairwise battle winning probabilities are exogenous by directly using the resulting matchings instead of strategy profiles. In this way, we also show that the expected 
winning probability of a team in the totally mixed equilibrium in Hamilton and Romano (1998) is the same as that where the contest organizer chooses a matching of players totally randomly (Proposition 1). Using this result, we show that the totally mixed strategy Nash equilibrium in Hamilton and Romano (1998) extends to a one-shot order choice game in the $\mathrm{Fu}, \mathrm{Lu}$, and Pan multi-battle contest environment in which each player's effort level is endogenously determined, and that the expected winning probability of a team is the same when the contest organizer chooses a matching of players totally randomly (Theorem 1). Although Fu, Lu, and Pan (2015) assume that the pairwise player matching in their multibattle contests is fixed, we show that their invariance result regarding the outcome (winning probability) of each pairwise battle is more general than that - as long as a pair of players are matched in one of the multiple battles in a team contest, the expected outcome (winning probability) stays the same, irrespective of the rest of the matches. Thus, for any realization of a matching as a result of (mixed strategy) equilibrium, the history independence result for the winning probability of each pairwise match in $\mathrm{Fu}, \mathrm{Lu}$, and, Pan (2015) still follows, resulting in the Hamilton-Romano totally random equilibrium.

More significantly, we extend the equivalence results in $\mathrm{Fu}, \mathrm{Lu}$, and Pan (2015) to a sequential battle-by-battle player-choice game. Here, the argument is much more involvedit is not a simple extension of sequential battles in Fu, Lu, and Pan (2015). At each subgame, the team leaders first choose players for the next battle, and then these players choose their effort levels. Thus, these players need to make a choice by foreseeing the outcomes in the subsequent subgames after the realization of the current battle's outcome. We will show, by backward induction arguments, that the team's ex ante winning probability in each subgame is the same as under the totally random matching of the remaining players by the contest organizer, thus its ex ante winning probability of the whole sequential battle-by-battle game is also the same as the ones under the Hamilton-Romano totally random Nash equilibrium in one-shot ordering choice game (Theorem 2). As a corollary, we can say that the ex ante expected equilibrium effort of each player is invariant of the type of player choice game - one- 
shot or sequential, since all matchings of players occur with the same ex ante probabilities in both equilibria. Thus, we add another invariance result to $\mathrm{Fu}, \mathrm{Lu}$, and Pan (2015).

In the next subsection, we provide a brief literature review. In Section 2, we will start with a three-battle contest example with exogenously fixed winning probabilities for each pairwise match between players from the two teams. This illustrates the equivalence between the outcome (ex ante team winning probability) of the one-shot game and that of the sequential move game. In Section 3, we introduce the general model using matching language and replicate Hamilton and Romano's (1998) result by using matching theory (Proposition 1). Then, in Section 4, we endogenize the winning probability of each race and show that the same results hold for both the one-shot and sequential ordering choice game (Theorems 1 and 2, and Corollary 1). In Section 5, we discuss the boundary of our result using several extensions and examples.

\subsection{Related Literature}

Our paper contributes to the burgeoning literature on multi-battle contests. ${ }^{3}$ Harris and Vickers (1987) model a two-firm R\&D competition as series of individual stages; in each stage the success probability depends on the firms' efforts in that stage. The first firm to win $N$ or more stages than its opponent wins the whole competition-a tug-of-war game. They show that the trailing firm makes less effort, and the effort decreases as the deficit increases - the momentum effect. Klumpp and Polborn (2006) consider a multi-district campaign spending game, e.g., US presidential primaries, in which district competitions are modeled as Tullock competitions and hold sequentially. The candidate who gets a majority of the districts wins the game. They show that the momentum effect exists and candidates tend to spend more in early voting districts. In Konrad and Kovenock (2009), two players compete in a race comprised of a sequence of battles, and each component battle is modeled as a first-price

\footnotetext{
${ }^{3}$ For a complete review of this literature, please see Kovenock and Roberson (2012).
} 
all-pay auction. They characterize the unique subgame perfect equilibrium and show that the expected effort in each battle can be non-monotonic as the competition gets tighter. All in all, both outcomes and strategies in each battle depend on the previous results. These history dependence results in this line of the literature are rooted in the presumption that the participants in each battle are the same.

There are papers other than $\mathrm{Fu}, \mathrm{Lu}$, and Pan (2015) that consider multi-battle group contests in which each battle is played by different players. Häfner (2017) investigates a tugof-war game played by a (potentially) infinite number of different players, and shows that there exists a unique Markov-perfect equilibrium. Barbieri and Serena (2019) show that the expected winners' efforts are higher if battles are held simultaneously than sequentially in the Fu-Lu-Pan model under a natural condition. These two papers assume that the matching of players is prefixed, but Barbieri and Serena (2019) also consider a contest-design problem and show that the sequential game in which players are ordered from less efficient to more efficient is the setup that minimizes winners' efforts. Fu and Lu (2018) consider a strategic player assignment game in a two-team, two-stage, all-pay contest, in which each team has one stronger and one weaker player. This model has the closest motivation to ours, but there is a fundamental difference between the two. They assume that the team with the higher aggregate effort wins the prize, whereas we assume that the team with the majority of individual battle victories wins. They show that in equilibrium, both teams assign the stronger players in the second stage as long as the intra-team heterogeneity of player ability is not excessive. Thus, it is easy to see that our neutrality result crucially depends on this difference. Klumpp, Konrad, and Solomon (2019) consider a sequential multi-battle Blotto game that respects the majoritarian rule, but where the resource is not reusable. ${ }^{4}$ They show that the player should split the resource evenly across all battles in the unique equilibrium,

\footnotetext{
${ }^{4}$ Konard (2018) also shows this even split result in a best-of-three contest. Konrad (2018) studies the best-of-three sequential Blotto game and shows that if the resource can be reused in future battles, there are discouragement effects for the lagging player and a showdown effect when the battle is decisive.
} 
and thus the winning chance in each battle is independent of how many games were won/lost before that battle.

\section{A Three-Player Example with Exogenous Winning Probabilities}

Here, we present a simple example that illustrates the equivalence between a one-shot ordering choice game and a battle-by-battle player choice game.

Example 1. Teams $A$ and $B$ each have three players labeled 1, 2, and 3. Suppose for simplicity that the winning probability in each pairwise battle is exogenously given. Since each match is a zero-sum game, we summarize these winning probabilities in a single matrix $(Q)$ from the perspective of team $A$ only:

$$
Q=\left(\begin{array}{lll}
q_{11} & q_{12} & q_{13} \\
q_{21} & q_{22} & q_{23} \\
q_{31} & q_{32} & q_{33}
\end{array}\right)
$$

where $q_{i j} \in[0,1]$ is the winning probability of team $A$ 's player $i$ when $i$ is matched with team $B$ 's player $j$ for all $i, j=1,2$, and 3 .

We first analyze the Nash equilibrium strategy profiles of the one-shot game in which both team leaders simultaneously choose the order in which their players compete. Note that each team leader's strategies are player orderings. For example, if leader $A$ plays 123 and leader $B$ plays 123 , then the resulting pairwise battles are $(1,1),(2,2)$, and $(3,3)$. Also, a strategy profile of 123 and 123 is the same as that of 132 and 132, and a strategy profile of 123 and 321 is the same as that of 132 and 312, etc. The winning probability of team $A$ for any strategy combination is, in principle, calculable. Therefore, we have the following 
payoff (winning probability) matrix for leader $A$ :

Leader $B$

\begin{tabular}{|c|c|c|c|c|c|c|c|}
\hline & 123 & 132 & 213 & 231 & 312 & 321 \\
\hline 123 & $\alpha$ & $\beta$ & $\gamma$ & $\delta$ & $\epsilon$ & $\phi$ \\
\cline { 2 - 8 } Leader $A$ & 132 & $\beta$ & $\alpha$ & $\delta$ & $\gamma$ & $\phi$ & $\epsilon$ \\
\cline { 2 - 8 } & 213 & $\gamma$ & $\epsilon$ & $\alpha$ & $\phi$ & $\beta$ & $\delta$ \\
\cline { 2 - 8 } & 231 & $\epsilon$ & $\gamma$ & $\phi$ & $\alpha$ & $\delta$ & $\beta$ \\
\hline 312 & $\delta$ & $\phi$ & $\beta$ & $\epsilon$ & $\alpha$ & $\gamma$ \\
\hline 321 & $\phi$ & $\delta$ & $\epsilon$ & $\beta$ & $\gamma$ & $\alpha$ \\
\hline
\end{tabular}

where, for example, $\alpha=q_{11} q_{22} q_{33}+q_{11} q_{22}\left(1-q_{33}\right)+\left(1-q_{11}\right) q_{22} q_{33}+q_{11}\left(1-q_{22}\right) q_{33}$ and $\beta, \gamma, \delta, \epsilon, \phi$ are similarly defined. Notice that $\alpha, \beta, \gamma, \delta, \epsilon, \phi$ show up exactly once for each row and column (though some of them may take the same values).

Now, assume that leader $B$ plays all pure strategies with probability $\frac{1}{6}$ each. Clearly, leader $A$ is indifferent between all pure strategies. Let leader $A$ play all pure strategies with probability $\frac{1}{6}$ each. Then, leader $B$ is also indifferent between all pure strategies. Thus, this is a mixed strategy equilibrium. Since this is a two-person zero-sum game, the Nash equilibrium payoff and the minimax value are the same. Moreover, by von Neumann's theorem, the minimax value is unique. Thus, we have unique Nash equilibrium winning probability $\bar{P}_{A}$, which is supported by a complete randomization with equal probabilities. This is the same result as in Hamilton and Romano (1998).

Notice that leader $A$ 's expected payoff is

$$
\bar{P}_{A}=\frac{\alpha+\beta+\gamma+\delta+\epsilon+\phi}{6}
$$

We now turn to a sequential choice game. That is, leader $A$ and leader $B$ simultaneously choose the first player, observe the outcome of the resulting match, and then choose their 
second player simultaneously (the players for the third battle are automatically determined using the leftover players). The question is what constitutes a subgame perfect equilibrium of this sequential game. We start with an analysis of each subgame. Suppose that in the first battle, team $A$ 's player 1 and team $B$ 's player 1 were matched and one of them won the first match. Whoever won, the rest of the game reduces to the order choice of the remaining two players on each team only. The resulting payoff matrix is as follows:

\begin{tabular}{c|c|c|c|}
\multicolumn{2}{c}{} & \multicolumn{1}{c}{$\frac{1}{2}$} & \multicolumn{1}{c}{} \\
\hline & & 23 & 32 \\
\cline { 2 - 4 }$\frac{1}{2}$ & 23 & $a$ & $b$ \\
\cline { 2 - 4 }$\frac{1}{2}$ & 32 & $b$ & $a$ \\
\cline { 2 - 5 } & &
\end{tabular}

In this matrix, $a, b \in[0,1]$ are team $A$ 's winning probabilities (strategy profiles $(23,23)$ and $(32,32)$ achieve the same winning probability, since players 2 and 2 and 3 and 3 are matched anyway). Notice that the unique Nash equilibrium in this zero-sum game is that both teams play 23 with probability $\frac{1}{2}$. This does not depend on which team won in the first match. Furthermore, the battle $(1,1)$ in the first round was chosen completely arbitrarily and the equal-probability continuation equilibrium is not affected. This means that in every subgame, both teams play $\frac{1}{2}$ and $\frac{1}{2}$ for the rest of the ordering no matter who was paired in the first battle and regardless of who wins it.

Now, we consider the first round battle. Suppose that leader $B$ selects players 1, 2, and 3 with probability $\frac{1}{3}$ for each. If leader $A$ chooses player 1 and leader $B$ happens to choose player 1 , leader $A$ knows that the subsequent battles $\{(2,2),(3,3)\}$ and $\{(2,3),(3,2)\}$ happen with an equal probability of $\frac{1}{2}$ for each. That is, the sets of pairwise battles $\{(1,1),(2,3),(3,2)\}$ and $\{(1,1),(2,2),(3,3)\}$ end up being played with probability $\frac{1}{3} \times \frac{1}{2}=\frac{1}{6}$ for each. The same argument applies to the case when leader $B$ happens to play 2 and 3 . In the end, each possible matching is played with probability $\frac{1}{6}$. Thus, leader $A$ is indifferent 
between choosing players 1,2 , or 3 in the first round, and in the second round he chooses the rest of the orderings with probability $\frac{1}{2}$ for each (this is equivalent to choosing a player from

the two remaining players with probability $\frac{1}{2}$ ). Clearly, leader $A$ will place probability $\frac{1}{3}$ for each of his three players in the first round. His equilibrium payoff is again $\bar{P}_{A}$. This discussion shows that the sequential game outcome is the same as the simultaneous game outcome. By induction, we can see that the argument works for any (odd) number of players.

\section{One-Shot Ordering Choice Game with Exogenous Winning Probabilities - the Hamilton-Romano Re- sult}

There are two teams, $A$ and $B$. Each team has $2 n+1$ players where $n \in N$. The whole competition consists of $2 n+1$ sequential (or simultaneous) head-to-head battles. The winning team is the one that wins $n+1$ battles. There is a team leader in charge of deciding the order in which players on each team will enjoy a payoff of 1 if his team wins. Let $N^{A}=\left\{i_{1}, \ldots, i_{2 n+1}\right\}$ and $N^{B}=\left\{j_{1}, \ldots, j_{2 n+1}\right\}$ be the sets of players of teams $A$ and $B$, and let $i$ and $j$ be the representative elements in $N^{A}$ and $N^{B}$, respectively. Team $v$ 's leader can choose the ordering of the players: $\pi^{A}:\{1, \ldots, 2 n+1\} \rightarrow N^{A}$ and $\pi^{B}:\{1, \ldots, 2 n+1\} \rightarrow N^{B}$ are oneto-one mappings. The two leaders announce the ordering of their players simultaneously at the beginning of the competition. Let $\Pi^{v}$ be the set of all orderings, and then a strategy combination is denoted by $\left(\pi^{A}, \pi^{B}\right) \in \Pi^{A} \times \Pi^{B}$.

Let a matching $\mu: N^{A} \rightarrow N^{B}$ be a one-to-one function such that $\mu^{-1}(\mu(i))=i$ for all $i \in N^{A}$. Since $\left|N^{A}\right|=\left|N^{B}\right|, \mu\left(N^{A}\right)=N^{B}$. Let $M\left(N^{A}, N^{B}\right)$ denote the set of all matchings. Note that there are $(2 n+1)$ ! possible matchings, and for each $\pi^{A}$, there is exactly one $\pi^{B}$ that generates a particular matching $\mu$. Moreover, given a matching $\mu$, there are $(2 n+1)$ ! combinations of $\left(\pi^{A}, \pi^{B}\right) \in \Pi^{A} \times \Pi^{B}$ that yield the same $\mu$. 
We assume that the winning probability of each match of players from teams $A$ and $B$ is independent of how other players are matched and which player wins. Team $A$ 's players' winning probabilities when they are matched with each of the players on team $B$ are exogenously given by ${ }^{5}$

$$
Q=\left(\begin{array}{ccc}
q_{i_{1} j_{1}} & \cdots & q_{i_{1} j_{2 n+1}} \\
\vdots & \ddots & \vdots \\
q_{i_{2 n+1} j_{1}} & \cdots & q_{i_{2 n+1} j_{2 n+1}}
\end{array}\right)
$$

where a generic match is denoted by $(i, j)$ with team $A$ 's $(i$ 's) winning probability being $q_{i j}$. This $Q$ matrix is perfectly general. We allow for the cases in which player $i_{1}$ does well against most of the players on team $B$, but $i_{1}$ somehow always loses against $j_{2 n+1}$.

The static nature of the winning probability matrix $Q$ implies that the payoffs of this game depend only on the resulting matching, i.e., two strategy profiles that lead to the same matching will result in identical payoffs for both teams. Denote the expected payoffs from a given matching for each team by $\tilde{P}^{A}(\mu)$ (and $\tilde{P}^{B}(\mu)=1-\tilde{P}^{A}(\mu)$ ) accordingly. Let $W=\left\{S \in 2^{\{1,2, \ldots, 2 n+1\}}:|S| \geq n+1\right\}$.

$$
\tilde{P}^{A}(\mu) \equiv \sum_{S \in W}\left(\prod_{r \in S}\left(q_{i_{r} \mu\left(i_{r}\right)}\right) \times \prod_{r \notin S}\left(1-q_{i_{r} \mu\left(i_{r}\right)}\right)\right) .
$$

There are $(2 n+1)$ ! strategy profiles $\left(\pi^{A}, \pi^{B}\right) \in \Pi^{A} \times \Pi^{B}$ that achieve the same matching $\mu \in M\left(N^{A}, N^{B}\right)$, where $M\left(N^{A}, N^{B}\right)$ denotes the set of all possible matchings. Also note that there are $(2 n+1)$ ! elements in $M$ and $((2 n+1) !)^{2}$ elements in $\Pi^{A} \times \Pi^{B}$. We now consider team $A$ 's winning probability when there exists a contest organizer who picks a

\footnotetext{
${ }^{5}$ In the next section, we endogenize winning probabilities in battles by considering a multi-battle contest game following $\mathrm{Fu}, \mathrm{Lu}$, and Pan (2015).
} 
matching totally randomly to be

$$
\bar{P}^{A} \equiv \frac{1}{(2 n+1) !} \sum_{\mu \in M\left(N^{A}, N^{B}\right)} \tilde{P}^{A}(\mu)
$$

Since the corresponding matching for any given combination of $\left(\pi^{A}, \pi^{B}\right)$ is unique, we can slightly abuse the notation to let $\mu: \Pi^{A} \times \Pi^{B} \rightarrow M\left(N^{A}, N^{B}\right)$ be the matching generated from permutations $\left(\pi^{A}, \pi^{B}\right)$, such that $\mu(i)=\pi^{B}\left(\left(\pi^{A}\right)^{-1}(i)\right)$ for all $i \in N^{A}$. Then, $A^{\prime}$ 's ex ante winning probability given by $\left(\pi^{A}, \pi^{B}\right)$ can be written as

$$
P^{A}\left(\pi^{A}, \pi^{B}\right) \equiv \tilde{P}^{A}\left(\mu\left(\pi^{A}, \pi^{B}\right)\right)
$$

Similarly, define $P^{B}\left(\pi^{A}, \pi^{B}\right)$. It is clear that $P^{A}\left(\pi^{A}, \pi^{B}\right)+P^{B}\left(\pi^{A}, \pi^{B}\right)=1$.

Thus, the game with two team leaders who maximize their teams' winning probability is a zero-sum game with strategy sets $\Pi^{A}$ and $\Pi^{B}$, and with a $\Pi^{A} \times \Pi^{B}$ payoff matrix $P \equiv\left(P^{A}\left(\pi^{A}, \pi^{B}\right)\right)_{\pi^{A} \in \Pi^{A}, \pi^{B} \in \Pi^{B}}$. In this case, a mixed strategy is $m^{v}: \Pi^{v} \rightarrow[0,1]$ with $\sum_{\pi^{v} \in \Pi^{v}} m^{v}\left(\pi^{v}\right)=1$ for $v=A, B$. Let $\bar{m}^{v}\left(\pi^{v}\right)=\frac{1}{\left|\Pi^{v}\right|}=\frac{1}{(2 n+1) !}$ for all $\pi^{v} \in \Pi^{v}$ and $k=A, B$ be the mixed strategy that assigns equal probability to all strategies. Notice that for each $A^{\prime}$ 's pure strategy $\pi^{A} \in \Pi^{A}$, each $\mu \in M\left(N^{A}, N^{B}\right)$ realizes once and only once for some $\pi^{B} \in \Pi^{B}$. With some abuse of notation, we have

$$
P^{A}\left(\pi^{A}, \bar{m}^{B}\right)=\frac{1}{(2 n+1) !} \sum_{\mu \in M\left(N^{A}, N^{B}\right)} \tilde{P}^{A}(\mu)=\bar{P}^{A}
$$

for any $\pi^{A} \in \Pi^{A}$, and team $A$ is indifferent between all possible orderings if team $B$ employs $\bar{m}^{B}$. For the same reason, team $B$ obtains payoff

$$
P^{B}\left(\bar{m}^{A}, \pi^{B}\right)=1-\bar{P}^{A}
$$


for any $\pi^{B} \in \Pi^{B}$. Therefore, we obtain the result by Hamilton and Romano (1998).

Proposition 1 (Hamilton and Romano 1998) Suppose that the winning probabilities of all pairwise battles are described by a static matrix $Q$. A total randomization over all orderings of players with equal probability $\left(\bar{m}^{A}, \bar{m}^{B}\right)$ is a Nash equilibrium of the one-shot orderingchoice game. Moreover, in every Nash equilibrium of the game, team A's winning probability, $\bar{P}^{A}$, is exactly the same as the one when the contest organizer picks a matching of players totally randomly.

Note that there are many other Nash equilibria in the one-shot ordering choice game, although the equilibrium payoffs are unique, as is shown in von Neumann (1928). For example, consider the following $2 n+1$ strategies: $\pi_{1}^{v}=\left(i_{1}, \ldots, i_{2 n+1}\right), \pi_{2}^{v}=\left(i_{2 n+1}, i_{1}, \ldots, i_{2 n}\right)$, $\pi_{2}^{v}=\left(i_{2 n}, i_{2 n+1}, i_{1}, \ldots, i_{2 n-1}\right), \ldots$, and $\pi_{2 n+1}^{v}=\left(i_{2}, \ldots, i_{2 n+1}, i_{1}\right)$. Let $\hat{m}^{v}$ be $\hat{m}^{v}\left(\pi_{\ell}^{v}\right)=\frac{1}{2 n+1}$ for all $\ell=1, \ldots, 2 n+1$ and $\hat{m}^{v}\left(\pi^{v}\right)=0$ for any other $\pi^{v}$. If team $B$ uses strategy $\hat{m}^{B}$, then each

player on team $A$ is matched with all of the team $B$ players with equal probability $\frac{1}{2 n+1}$. Thus, team $A$ is indifferent between all strategies in $\Pi^{A}$. Therefore, $\hat{m}^{A}$ is one of the best responses to $\hat{m}^{B}$, and $\left(\hat{m}^{A}, \hat{m}^{B}\right)$ is a Nash equilibrium, too. There are many other ways to select $2 n+1$ pure strategies that do this same thing. Hence, we have a continuum of Nash equilibria with the same expected payoffs.

\section{The Main Analysis - Endogenous Winning Probabil- ities}

\subsection{The One-Shot Order Choice Game}

So far, we have assumed that the winning probabilities for team $A$ 's players against team $B$ 's players are exogenously determined for all possible pairs of player matches, i.e., players' 
behavior is exogenous. In this section, we relax this assumption following the arguments in $\mathrm{Fu}, \mathrm{Lu}$, and Pan (2015). We again assume that $\left|N^{A}\right|=\left|N^{B}\right|=2 n+1$ and that the leaders of teams $A$ and $B$ simultaneously choose the player ordering at the beginning of the contest. Consider a battle between players $i \in N^{A}$ and $j \in N^{B}$. Although the same result applies to any of the examples listed in their paper, we will focus on a variation of a complete-information generalized Tullock contest (Model 6 in Fu, Lu, and Pan 2015). To apply their invariance result, assume that (ij-pair-specific) contest success function $q_{i j}\left(x_{i}, x_{j}\right)$ is (i) homogenous of degree zero in $x_{i}$ and $x_{j}$, (ii) $\frac{\partial q_{i j}}{\partial x_{i}}>0$ and $\frac{\partial^{2} q_{i j}}{\partial x_{i}^{2}}<0$, and (iii) $\frac{\partial q_{i j}}{\partial x_{j}}<0$ and $\frac{\partial^{2} q_{i j}}{\partial x_{j}^{2}}>0$, where $x_{i}$ and $x_{j}$ are effort levels by players $i$ and $j$, respectively. Players $i$ and $j$ have constant marginal costs of effort $c_{i}, c_{j}>0$ and benefits $V_{i}, V_{j}>0$ from their team's winning the majority of battles. If this is just a single battle played by $i$ and $j$, then players $i$ and $j$ solve the following problems, respectively:

$$
\max _{x_{i}} q_{i j}\left(x_{i}, x_{j}\right) V_{i}-c_{i} x_{i}
$$

and

$$
\max _{x_{j}}\left(1-q_{i j}\left(x_{i}, x_{j}\right)\right) V_{j}-c_{j} x_{j}
$$

The following result is first shown by Malueg and Yates (2005). For completeness, we include a concise proof. ${ }^{6}$

Lemma 1 (Malueg and Yates 2005) In a complete-information general Tullock contest played by $(i, j)$, team A member $i$ 's equilibrium winning probability $i s \bar{q}_{i j}=q_{i j}\left(\frac{c_{j}}{V_{j}}, \frac{c_{i}}{V_{i}}\right)$. Moreover, if the equilibrium effort vector given a prize is $\left(x_{i}^{*}(i, j), x_{j}^{*}(i, j)\right)$, then the equilibrium effort vector is $\left(p x_{i}^{*}(i, j), p x_{j}^{*}(i, j)\right)$ when the size of the prize is multiplied by $p$; i.e., the equilibrium efforts are homogeneous of degree one in the value of the prize.

${ }^{6}$ This is a variation on Observations 1 and 2 in Fu, Lu, and Pan (2015) in our context. In their Extensions and Caveats section, they show that asymmetric valuations can be allowed as long as there is no personal battle-specific payoff. 
Proof. The first order conditions are

$$
\frac{\partial q_{i j}\left(x_{i}, x_{j}\right)}{\partial x_{i}} V_{i}-c_{i}=0
$$

and

$$
-\frac{\partial q_{i j}\left(x_{i}, x_{j}\right)}{\partial x_{j}} V_{j}-c_{j}=0
$$

Since $q_{i j}\left(x_{i}, x_{j}\right)$ is homogenous of degree zero, we have a Euler equation

$$
\frac{\partial q_{i j}\left(x_{i}, x_{j}\right)}{\partial x_{i}} x_{i}+\frac{\partial q_{i j}\left(x_{i}, x_{j}\right)}{\partial x_{j}} x_{j}=0
$$

These three equations imply

$$
\frac{x_{i}}{x_{j}}=\frac{V_{i} c_{j}}{V_{j} c_{i}}
$$

Thus, team $A$ 's equilibrium winning probability is written as

$$
\bar{q}_{i j}=q_{i j}\left(\frac{V_{i}}{c_{i}}, \frac{V_{j}}{c_{j}}\right)
$$

Since $q_{i j}\left(x_{i}, x_{j}\right)$ is homogenous of degree zero, $\frac{\partial q_{i j}\left(x_{i}, x_{j}\right)}{\partial x_{i}}$ and $\frac{\partial q_{i j}\left(x_{i}, x_{j}\right)}{\partial x_{j}}$ are homogeneous of degree - 1 . Thus, we have

$$
\frac{\partial q_{i j}\left(p x_{i}, p x_{j}\right)}{\partial\left(p x_{i}\right)}=\frac{1}{p} \frac{\partial q_{i j}\left(x_{i}, x_{j}\right)}{\partial x_{i}}
$$

for all $p>0$ (the same result holds for $x_{j}$ ). This implies

$$
\frac{\partial q_{i j}\left(p x_{i}, p x_{j}\right)}{\partial\left(p x_{i}\right)} p V_{i}-c_{i}=\frac{\partial q_{i j}\left(x_{i}, x_{j}\right)}{\partial x_{i}} V_{i}-c_{i}=0 .
$$

That is, if $\left(x_{i}, x_{j}\right)=\left(x_{i}^{*}(i, j), x_{j}^{*}(i, j)\right)$ solves the system of equations (1) and (2), then 
$\left(x_{i}, x_{j}\right)=\left(p x_{i}^{*}(i, j), p x_{j}^{*}(i, j)\right)$ solves the system of equations

$$
\frac{\partial q_{i j}\left(x_{i}, x_{j}\right)}{\partial x_{i}} p V_{i}-c_{i}=0
$$

and

$$
-\frac{\partial q_{i j}\left(x_{i}, x_{j}\right)}{\partial x_{j}} p V_{j}-c_{j}=0 .
$$

We have completed the proof.

Thus, as long as conditions (i), (ii), and (iii) are satisfied, the winning probability of player $i$ in a battle with player $j$ is intact at $\bar{q}_{i j}$, since players $i$ and $j$ face the same probability of their battle to be pivotal $p$ in every contest with multiple pairwise battles. This is the Observation 2 in Fu, Lu, and Pan (2015). Denote $\bar{Q}\left(N^{A}, N^{B}\right)=\left(\bar{q}_{i j}\right)_{i \in N^{A}, j \in N^{B}}$ to be the pairwise winning probability of player $i$ on team $A$ against $j$ on team $B$. Thus, the winning probability of team $A$ in a multi-battle contest under fixed matching $\mu$ is always described by

$$
\tilde{P}^{A}(\mu) \equiv \sum_{S \in W}\left(\prod_{r \in S}\left(\bar{q}_{i_{r} \mu\left(i_{r}\right)}\right) \times \prod_{r \notin S}\left(1-\bar{q}_{i_{r} \mu\left(i_{r}\right)}\right)\right) .
$$

Using this, we immediately get the following result.

Theorem 1 (one-shot order choice game). In a multi-battle generalized Tullock contest, suppose that the two teams simultaneously choose the order in which their players will fight in the battles. We have the following: (i) for any realized matching $\mu$ and any pair $(i, j)$ with $\mu(i)=j$, player $i$ 's winning probability is invariant at $\bar{q}_{i j}$, and (ii) a totalrandomization strategy profile-both players' placing probability $\frac{1}{(2 n+1) !}$ in all orderingsis a mixed strategy Nash equilibrium; and (iii) team A's expected winning probability is $\bar{P}^{A}=\frac{1}{(2 n+1) !} \sum_{\mu \in M} \tilde{P}^{A}(\mu)$.

Proof. By Observations 1 and 2 in Fu, Lu, and Pan (2015) and Lemma 1, we know that for 
any realized matching $\mu \in M\left(N^{A}, N^{B}\right)$, in any battle by matched players $(i, j)$ with $\mu(i)=j$, team $A$ wins with probability $\bar{q}_{i j}$. Thus, team $A$ 's winning probability matrix is $\bar{Q}\left(N^{A}, N^{B}\right)$. This implies that by Proposition 1, (ii) and (iii) must hold.

\subsection{The Battle-by-Battle Player Choice Game}

Now, we will consider sequential battle-by-battle player-choice games. Consider a state $s \in S$ with $s=\left(k, \ell, h ; T^{A}, T^{B}\right)$, where $k$ is number of battles left, and $\ell$ and $h$ denote the number of wins that teams $A$ and $B$ need to become the winning team at state $s$, respectively. Moreover, $T^{A}$ and $T^{B}$ denote the set of remaining players for teams $A$ and $B$, respectively, and $S$ is the set of all states. Note that $k=\left|T^{A}\right|=\left|T^{B}\right|$ and $\ell+h=k+1$. We use the functions $k(s)=k, \ell(s)=\ell, h(s)=s, T^{A}(s)=T^{A}$, and $T^{B}(s)=T^{B}$ to indicate the relevant information at state $s=\left(k, \ell, h ; T^{A}, T^{B}\right)$. We start with the following definition. In state $s$, let

$$
\bar{P}^{A}(s) \equiv \frac{1}{k(s) !} \sum_{\mu \in M\left(T^{A}(s), T^{B}(s)\right)} \tilde{P}(\mu ; k(s), \ell(s))
$$

where

$$
\tilde{P}(\mu ; k, \ell) \equiv \sum_{S \in W(k, \ell)}\left(\prod_{r \in S}\left(\bar{q}_{i_{r} \mu\left(i_{r}\right)}\right) \times \prod_{r \notin S}\left(1-\bar{q}_{i_{r} \mu\left(i_{r}\right)}\right)\right)
$$

and

$$
W(k, \ell) \equiv\left\{S \in 2^{\{1, \ldots, k\}}:|S| \geq \ell\right\}
$$

Note that $W(k, \ell)$ is the set of winning coalitions when a team needs to win $\ell$ out of $k$ battles. Similar to the previous section, $\bar{P}^{A}(s)$ is $A^{\prime}$ 's winning probability when there is a contest organizer who totally randomly assigns players to battles after the state $s$. We let $\triangle\left(T^{A}(s)\right)$ and $\triangle\left(T^{B}(s)\right)$ be the sets of mixed actions for leader $A$ and $B$, respectively, and define $\sigma^{v}: S \rightarrow \triangle\left(N^{v}\right)$ such that $\sigma^{v}(s) \in \triangle\left(T^{v}(s)\right)$ as the mixed strategy of the leader $v$. One possible subgame perfect equilibrium strategy is $\bar{\sigma}^{v}(s)=\frac{1}{\left|T^{v}(s)\right|}(1,1, \ldots, 1) \in \triangle T^{v}(s)$ for 
$v=A, B$.

In each state $s$, we need to consider every possible pair of players in the next battle. For each pair, $i \in T^{A}(s)$ and $j \in T^{B}(s)$, depending on the winner of the battle, the next state will be either $s_{-i j}^{i}=\left(k-1, \ell-1, h ; T^{A} \backslash\{i\}, T^{B} \backslash\{j\}\right)$ or $s_{-i j}^{j}=\left(k-1, \ell, h-1 ; T^{A} \backslash\{i\}, T^{B} \backslash\{j\}\right)$. The former $s_{-i j}^{i}$ denotes the state that succeeds $s$ after a battle between $i$ and $j$ where $i$ wins. Furthermore, we will prove the following result using induction arguments starting from the last battle.

Theorem 2 (battle-by-battle player choice game). In a multi-battle generalized Tullock contest, suppose that the two teams simultaneously choose their players battle by battle sequentially. Then, we have the following: (i) in any battle in any stage, if players $i \in T^{A}$ and $j \in T^{B}$ are matched, i's winning probability is invariant at $\bar{q}_{i j}$; (ii) the total-randomization strategy profile $\left(\bar{\sigma}^{A}, \bar{\sigma}^{B}\right)$ is a subgame-perfect equilibrium; and (iii) team A's expected winning probability in the beginning of each state $s \in S$ is $\bar{P}^{A}(s)$. In particular, for the initial state $s_{0}$, we have $\bar{P}^{A}\left(s_{0}\right)=\bar{P}^{A}=\frac{1}{(2 n+1) !} \sum_{\mu \in M} \tilde{P}^{A}(\mu)$.

Proof. By induction, we will show that for any state $s$ with $k(s) \leq \hat{k}$ with (i)-(iii) satisfied, then for a state $s^{\prime}$ with $k(s)=\hat{k}+1$, (i)-(iii) are again satisfied.

Suppose that $\hat{k}=1$. For any state $s$ with $k(s)=1$, the only meaningful case is $k=\ell=$ $h=1$ (otherwise, the game is over). Clearly, the last players $i$ and $j$ make the best effort to obtain the award $V_{i}$ and $V_{j}$, respectively, so the winning probability of team $A$ is $\bar{q}_{i j}$. In any other case, the game is over. Moreover, (ii) and (iii) in this case are trivial.

Now, suppose that $\hat{k}=2$. There are two meaningful cases: $(k, \ell, h)=(2,2,1)$ or $(2,1,2)$. Consider $(k, \ell, h)=(2,2,1)$. Let $T^{A}=\left\{i, i^{\prime}\right\}$ and $T^{B}=\left\{j, j^{\prime}\right\}$. We know that if the game is not over after this round (team $A$ player wins), then team $A$ 's winning probability is $q_{i^{\prime} j^{\prime}}$ if $\left\{i^{\prime}, j^{\prime}\right\}$ is selected in the subgame. What, then, about the second last stage played by players 
$i$ and $j$ ? The payoff functions of players $i$ and $j$ are given as

$$
\left[q_{i j}\left(x_{i}, x_{j}\right) \bar{q}_{i^{\prime} j^{\prime}}\right] V_{i}-c_{i} x_{i}
$$

and

$$
\left[1-q_{i j}\left(x_{i}, x_{j}\right) \bar{q}_{i^{\prime} j^{\prime}}\right] V_{j}-c_{j} x_{j}
$$

respectively. The first order conditions are

$$
\frac{\partial q_{i j}\left(x_{i}, x_{j}\right)}{\partial x_{i}} \bar{q}_{i^{\prime} j^{\prime}} V_{i}-c_{i}=0
$$

and

$$
-\frac{\partial q_{i j}\left(x_{i}, x_{j}\right)}{\partial x_{j}} \bar{q}_{i^{\prime} j^{\prime}} V_{j}-c_{j}=0
$$

Thus, $\frac{x_{i}}{x_{j}}=\frac{V_{i} c_{j}}{V_{j} c_{i}}$ and $q_{i j}\left(\frac{c_{j}}{V_{j}}, \frac{c_{i}}{V_{i}}\right)=\bar{q}_{i j}$. The matrix game of this subgame is described by

\begin{tabular}{c|c|c|c|}
\multicolumn{2}{c}{} & \multicolumn{1}{c}{$\frac{1}{2}$} & \multicolumn{1}{c}{$\frac{1}{2}$} \\
\cline { 2 - 4 } \multicolumn{1}{c|}{$\ell=2$} & $j j^{\prime}$ & $j^{\prime} j$ \\
\cline { 2 - 4 }$\frac{1}{2}$ & $i i^{\prime}$ & $\bar{q}_{i j} \bar{q}_{i^{\prime} j^{\prime}}$ & $\bar{q}_{i j^{\prime}} \bar{q}_{i^{\prime} j}$ \\
\cline { 2 - 4 }$\frac{1}{2}$ & $i^{\prime} i$ & $\bar{q}_{i^{\prime} j^{\prime}} \bar{q}_{i^{\prime} j}$ & $\bar{q}_{i j} \bar{q}_{i^{\prime} j^{\prime}}$ \\
\cline { 2 - 5 } & & &
\end{tabular}

Clearly, a mixed strategy profile with equal probability, $\left(\bar{\sigma}^{A}(s), \bar{\sigma}^{B}(s)\right)$, is an equilibrium and is unique unless $\bar{q}_{i j} \bar{q}_{i^{\prime} j^{\prime}}=\bar{q}_{i j} \bar{q}_{i^{\prime} j^{\prime}}$. Team $A$ 's winning probability (expected payoff) is $\frac{1}{2}\left(\bar{q}_{i j} \bar{q}_{i^{\prime} j^{\prime}}+\bar{q}_{i j^{\prime}} \bar{q}_{i^{\prime} j}\right)=\bar{P}^{A}\left(T^{A}, T^{B}, 2,2,1\right)$. Case $(k, \ell, h)=(2,1,2)$ can be treated symmetrically by swapping teams $A$ and $B$. This proves that the induction hypothesis holds for $k=2$.

Consider any subgame starting at state $s=\left(k, \ell, h ; T^{A}, T^{B}\right)$ with $\left|T^{A}\right|=\left|T^{B}\right|=k$ and suppose that the induction hypothesis is correct for all states $\tilde{s}=\left(\tilde{k}, \tilde{\ell}, \tilde{h} ; \tilde{T}^{A}, \tilde{T}^{B}\right)$ with $\left|\tilde{T}^{A}\right|=\left|\tilde{T}^{B}\right|=\tilde{k}$ where $\tilde{k}<k$. Denote the set of all possible matchings between the 
members of $T^{A}$ and $T^{B}$ by $M\left(T^{A}, T^{B}\right)$. Similarly, denote the set of all possible matchings between the members of $T^{A}$ and $T^{B}$ in which player $i \in T^{A}$ is matched to player $j \in T^{B}$ by $M\left(T^{A}, T^{B} ;(i, j)\right)$. Then, the continuation state when player $i$ wins is $s_{-i j}^{i}=(k-1, \ell-$ $\left.1, h ; T^{A} \backslash\{i\}, T^{B} \backslash\{j\}\right)$ and when $j$ wins the state is $s_{-i j}^{j}=\left(k-1, \ell, h-1 ; T^{A} \backslash\{i\}, T^{B} \backslash\{j\}\right)$. We first show that (i) holds for any $s$ with $k(s)=k$. The payoff functions of players $i$ and $j$ after being matched in state $s$ are

$$
\begin{aligned}
u_{i} & =q_{i j}\left(x_{i}, x_{j}\right) \bar{P}^{A}\left(s_{-i j}^{i}\right) V_{i}+\left(1-q_{i j}\left(x_{i}, x_{j}\right)\right) \bar{P}^{A}\left(s_{-i j}^{j}\right) V_{i}-c_{i} x_{i} \\
& =q_{i j}\left(x_{i}, x_{j}\right)\left(\bar{P}^{A}\left(s_{-i j}^{i}\right)-\bar{P}^{A}\left(s_{-i j}^{j}\right)\right) V_{i}-c_{i} x_{i}+\bar{P}^{A}\left(s_{-i j}^{j}\right) V_{i}
\end{aligned}
$$

and

$$
\begin{aligned}
u_{j} & =\left(1-q_{i j}\left(x_{i}, x_{j}\right)\right)\left(1-\bar{P}^{A}\left(s_{-i j}^{j}\right)\right) V_{j}+q_{i j}\left(x_{i}, x_{j}\right)\left(1-\bar{P}^{A}\left(s_{-i j}^{i}\right)\right) V_{j}-c_{j} x_{j} \\
& =\left(1-q_{i j}\left(x_{i}, x_{j}\right)\right)\left(\bar{P}^{A}\left(s_{-i j}^{i}\right)-\bar{P}^{A}\left(s_{-i j}^{j}\right)\right) V_{j}-c_{j} x_{j}-\left(1-\bar{P}^{A}\left(s_{-i j}^{i}\right)\right) V_{j},
\end{aligned}
$$

respectively. Thus, by our Lemma 1, equilibrium efforts $\left(x_{i}, x_{j}\right)$ satisfy $\frac{x_{i}}{x_{j}}=\frac{V_{i} c_{j}}{V_{j} c_{i}}$ and team $A$ 's winning probability is invariant at $q_{i j}\left(x_{i}, x_{j}\right)=\bar{q}_{i j}$.

Now, let team leader $B$ randomize his choice of player at state $s$ with equal probability, i.e., he uses $\bar{\sigma}^{B}(s)=\frac{1}{k(s)}$. The resulting winning probability for team $A$ from selecting player $i$ at state $s$ is: 


$$
\begin{aligned}
& \frac{1}{k} \sum_{j \in T^{B}}\left[\bar{q}_{i j} \bar{P}^{A}\left(s_{-i j}^{i}\right)+\left(1-\bar{q}_{i j}\right) \bar{P}^{A}\left(s_{-i j}^{j}\right)\right] \\
& =\frac{1}{k} \sum_{j \in T^{B}}\left[\bar{q}_{i j} \frac{1}{(k-1) !} \sum_{\mu \in M\left(T^{A}\left(s_{-i j}^{i}\right), T^{B}\left(s_{-i j}^{i}\right)\right)} \tilde{P}(\mu, k-1, l-1)\right] \\
& +\frac{1}{k} \sum_{j \in T^{B}}\left[\left(1-\bar{q}_{i j}\right) \frac{1}{(k-1) !} \sum_{\mu \in M\left(T^{A}\left(s_{-i j}^{j}\right), T^{B}\left(s_{-i j}^{j}\right)\right)} \tilde{P}(\mu, k-1, l)\right] \\
& =\frac{1}{k !} \sum_{j \in T^{B}} \sum_{\mu \in M\left(T^{A}\left(s_{-i j}^{i}\right), T^{B}\left(s_{-i j}^{i}\right)\right)} \bar{q}_{i j} \tilde{P}(\mu, k-1, l-1) \\
& +\frac{1}{k !} \sum_{j \in T^{B}} \sum_{\mu \in M\left(T^{A}\left(s_{-i j}^{j}\right), T^{B}\left(s_{-i j}^{j}\right)\right)}\left(1-\bar{q}_{i j}\right) \tilde{P}(\mu, k-1, l) \\
& =\frac{1}{k !} \sum_{j \in T^{B}} \sum_{\mu \in M\left(T^{A}-\{i\}, T^{B}-\{j\}\right)}\left[\bar{q}_{i j} \tilde{P}(\mu, k-1, l-1)+\left(1-\bar{q}_{i j}\right) \tilde{P}(\mu, k-1, l)\right] \\
& =\sum_{j \in T^{B}} \frac{1}{k}\left[\frac{1}{(k-1) !} \sum_{\mu \in M\left(T^{A}, T^{B} ;(i, j)\right)} \tilde{P}(\mu, k, l)\right]=\bar{P}^{A}(s)
\end{aligned}
$$

where $M\left(T^{A}, T^{B} ;(i, j)\right)$ is a collection of all matchings $\mu: T^{A} \rightarrow T^{B}$ with $\mu(i)=j$.

Each term inside the brackets in the last line equation is the ex ante probability that team $A$ wins the tournament when player $i$ faces player $j$, but since we average them out over all $j \in T^{B}$, the RHS is equal to the overall probability of winning the tournament under the assumption that the leader of team $B$ mixes equally among all players. We know this since each battle's winning probability is independent of other battles' outcomes from Lemma 1. Note that from the inductive assumption mixing equally is a subgame perfect equilibrium in every subsequent state of the world, and thus it follows that each subgame is weighted by 
the same probability $\frac{1}{(k-1) !}$.

Clearly, the winning probability of team $A$ is $\bar{P}^{A}(s)$, regardless of which player $i \in T^{A}(s)$ is chosen by team $A$ at state $s$. Thus, team $A$ is indifferent between all available players. Team $A$ can place equal probability on each player, which makes team $B$ indifferent between all available players. This concludes that team $A$ 's equilibrium winning probability at state $s$ is $\bar{P}^{A}(s)$, which is team $A$ 's winning probability when every possible match occurs with equal probability. Our induction argument is complete.

Note that at the initial state $s_{0}=\left(2 n+1, n+1, n+1 ; N^{A}, N^{B}\right)$, we have $\bar{P}^{A}\left(s_{0}\right)=\bar{P}^{A}=$ $\frac{1}{(2 n+1) !} \sum_{\mu \in M} \tilde{P}^{A}(\mu)$ by definition.

Next, we turn to each player's ex ante expected effort. By Theorem 2, we know that every matching $\mu$ occurs with probability $\frac{1}{(2 n+1) !}$ ex ante. Since the winning probability matrix $\bar{Q}$ is independent according to Theorems 1 and 2, we can calculate the probability that $i$ and $j$ are matched at state $s=\left(k, \ell, h ; T^{A}, T^{B}\right)$, with this battle being pivotal. First, state $s$ occurs with many possible matchings prior to the battle -in all elements $\tilde{\mu} \in M\left(N^{A} \backslash T^{A}(s), N^{B} \backslash T^{B}(s)\right)$. Second, player $i$ is matched with player $j$ with probability $\frac{1}{k(s)}$. Third, after the battle $(i, j)$ is over, there are many possible matching realizations $\left.\mu\right|_{\left(T^{A} \backslash\{i\}, T^{B} \backslash\{j\}\right)} \in M\left(T^{A}(s) \backslash\{i\}, T^{B}(s) \backslash\{j\}\right)$ with probability $\frac{1}{(k(s)-1) !}$ for each. Fourth, for each possible matching realization $\hat{\mu} \in M\left(T^{A} \backslash\{i\}, T^{B} \backslash\{j\}\right)$, the probability that this $(i, j)$ battle is pivotal is

$$
p(s,(i, j))=\sum_{\hat{\mu} \in M\left(T^{A}(s) \backslash\{i\}, T^{B}(s) \backslash\{j\}\right)} \sum_{S \in D(k(s)-1, \ell(s)-1)} \prod_{r \in S}\left(\bar{q}_{i_{r} \hat{\mu}\left(i^{r}\right)}\right) \times \prod_{r \notin S}\left(1-\bar{q}_{i_{r} \hat{\mu}\left(i_{r}\right)}\right),
$$

where $D(k, \ell) \equiv\{S \subseteq\{1, \ldots, k\}:|S|=\ell\}$. Since this probability is common to players $i$ and $j$, player $i$ 's expected effort when $i$ and $j$ are matched is $p(s,(i, j)) x_{i}^{*}(i, j)$ by Lemma 1 . Since 
state $s$ occurs with probability

$$
P(s)=\sum_{\tilde{\mu} \in M\left(N^{A} \backslash T^{A}(s), N^{B} \backslash T^{B}(s)\right)} \sum_{S \in D(2 n+1-k(s), n+1-\ell(s))} \prod_{r \in S}\left(\bar{q}_{i_{r} \tilde{\mu}\left(i^{r}\right)}\right) \times \prod_{r \notin S}\left(1-\bar{q}_{i_{r} \tilde{\mu}\left(i_{r}\right)}\right),
$$

player $i$ 's expected effort when $i$ is matched with $j$ is

$$
\begin{aligned}
E\left(x_{i} \mid(i, j)\right) & =\sum_{s \in \mathcal{S} \mid(i, j) \in T^{A}(s) \times T^{B}(s)} P(s) p(s,(i, j)) x_{i}^{*}(i, j) \\
& =\sum_{\tilde{\mu} \in M\left(N^{A} \backslash\{i\}, N^{B} \backslash\{j\}\right)} \sum_{S \in D(2 n, n)} \prod_{r \in S}\left(\bar{q}_{i_{r} \tilde{\mu}\left(i^{r}\right)}\right) \times \prod_{r \notin S}\left(1-\bar{q}_{i_{r} \tilde{\mu}\left(i_{r}\right)}\right) x_{i}^{*}(i, j) .
\end{aligned}
$$

Thus, the coefficient of $x_{i}^{*}(i, j)$ is nothing but the probability that this battle becomes pivotal. This implies that neither a sequential choice nor a one-shot choice makes a difference. Hence, player $i$ 's ex ante expected effort in both cases is

$$
\begin{aligned}
E\left(x_{i}\right) & =\frac{1}{2 n+1} \sum_{j \in N^{B}} E\left(x_{i} \mid(i, j)\right) \\
& =\frac{1}{2 n+1} \sum_{j \in N^{B}} \sum_{\tilde{\mu} \in M\left(N^{A} \backslash\{i\}, N^{B} \backslash\{j\}\right)} \sum_{S \in\left\{S^{\prime} \in\{1, \ldots, 2 n\}:\left|S^{\prime}\right|=n\right\}} \prod_{r \in S}\left(\bar{q}_{i_{r} \tilde{\mu}\left(i^{r}\right)}\right) \times \prod_{r \notin S}\left(1-\bar{q}_{i_{r} \tilde{\mu}\left(i_{r}\right)}\right) x_{i}^{*}(i, j),
\end{aligned}
$$

and Fu, Lu, and Pan's (2015) total effort equivalence result extends to our case, too.

Corollary 1. The expected effort level of each player in a one-shot ordering choice game is equal to the level in a battle-by-battle sequential choice game.

Although we only considered a fully sequential player-choice game in Theorem 2, Fu, Lu, and Pan's (2015) invariance results hold even if the game involves battles with a more general temporal structure, although the argument gets messier by that (see Appendix for a formal analysis). 


\section{$5 \quad$ Robustness and Subtleties in Our Results}

Here, we consider possible extensions of our model to see the boundaries of our invariance results. It turns out that the choices of player orderings often add more subtleties for the results on the expected winning probability of the whole contest and ex ante effort levels.

\subsection{Private Benefits from Winning Battles}

We start with a positive result in an extension discussed in Fu, Lu, and Pan (2015). First, we consider the case where players get private benefits from winning their battle in addition to their team's winning the prize. Let players $i$ and $j$ get $\pi_{i j}^{i}$ and $\pi_{i j}^{j}$ from winning battle $(i, j)$. Then, players $i$ and $j$ 's gross benefits $\tilde{V}^{i}$ and $\tilde{V}^{j}$ are written as

$$
\begin{aligned}
\tilde{V}^{i} & =\pi_{i j}^{i}+p(i, j) V^{i} \\
\tilde{V}^{j} & =\pi_{i j}^{j}+p(i, j) V^{j}
\end{aligned}
$$

where $p_{i j}>0$ denotes the probability that battle $(i, j)$ becomes pivotal. Since the above equalities need to hold for any $p(i, j)$, by Lemma 1 , we can say that the following holds

$$
\frac{\tilde{V}^{i}}{\tilde{V}^{j}}=\frac{V^{i}}{V^{j}}=\frac{\pi_{i j}^{i}}{\pi_{i j}^{j}}
$$

for all $i$ and $j$. This implies that equilibrium $\bar{q}_{i j}$ is constant as long as there is a common $\gamma_{i j}>0$ for any battle $(i, j)$ such that $\pi_{i j}^{i}=\gamma_{i j} V^{i}$ and $\pi_{i j}^{j}=\gamma_{i j} V^{j}$ hold. Note that $\gamma_{i j}$ s do not need to be the same for different $(i, j)$ s. With this property, all equivalence results in Theorems 1 and 2 and Corollary 1 still hold. 


\subsection{Heterogeneous Weights}

Unlike in Fu, Lu, and Pan (2015), our player-order choice game does not preserve the invariance in a team's winning probability if battles are weighted unevenly. In the last section of Fu, Lu, and Pan (2015), they demonstrate the robustness of invariance results that allow for component battles to carry different weights. This result follows in their model, since each battle and the players who play in them are tied up together. However, in our game, team leaders assign players to each battle. If a certain battle is weighted heavily, team leaders' strategy would be affected - if the next battle is pivotal due to its heavy weight, the team leaders do not totally randomize - they want to assign the strongest players to this key battle.

Example 2. Suppose that there are three battles with potentially different weights, and teams $A$ and $B$ have three players each. The team that wins with a total weight of more than $\frac{1}{2}$ wins the contest. We assume the following exogenous probability matrix:

$$
Q=\left(\begin{array}{lll}
q_{11} & q_{12} & q_{13} \\
q_{21} & q_{22} & q_{23} \\
q_{31} & q_{32} & q_{33}
\end{array}\right)=\left(\begin{array}{lll}
0.5 & 0.7 & 0.9 \\
0.3 & 0.5 & 0.7 \\
0.1 & 0.3 & 0.5
\end{array}\right)
$$

Player 1 is the dominant player on each team. If the weight of each battle is $\frac{1}{3}$ each as before, then we know that the total randomization is used in any setup. But now, suppose that the first battle's weight is more than $\frac{1}{2}$. In this case, only the first battle matters for the contest outcome. Obviously, both team leaders assign their best player to the first battle. Thus, our results no longer hold. 


\subsection{Excess Players}

Note that we have been assuming that the number of players who participate in the $2 n+1$ battles from each team needs to be exactly $2 n+1$. Although this assumption is natural in $\mathrm{Fu}, \mathrm{Lu}$, and Pan (2015), it is essential for our equivalence results as we can see from the following example. For simplicity, we consider a game with an exogenous winning probability matrix again.

Example 3. Suppose that there are three battles and teams $A$ and $B$ have four and three players, respectively. We assume the following exogenous probability matrix:

$$
Q=\left(\begin{array}{lll}
q_{11} & q_{12} & q_{13} \\
q_{21} & q_{22} & q_{23} \\
q_{31} & q_{32} & q_{33} \\
q_{41} & q_{42} & q_{43}
\end{array}\right)=\left(\begin{array}{ccc}
0 & 0.5 & 0.5 \\
0 & 0.5 & 0.5 \\
0 & 0.5 & 0.5 \\
0.5 & 0 & 0
\end{array}\right)
$$

That is, player 1 on team $B$ is a dominant player, but players 1,2 , and 3 on team $A$ and players 2 and 3 on team $B$ are in the exact same league. Player 4 on team $A$ is a weak player, but is good at dealing with the dominant player 1 on team $B$ (an assassin). In this case, if team $A$ selects $\{1,2,3\}$, team $A$ can win only when both players that are not matched with team $B$ 's dominant player win. Thus, team $A$ 's winning probability is $0.5 \times 0.5=0.25$. If team $A$ includes the assassin player 4 , then it has a positive winning probability only when the assassin player is matched with the dominant player. This implies that team $A$ 's winning probability is $\frac{1}{3} \times 0.5=0.1333<0.25$. Thus, in a one-shot static ordering choice game, team $A$ does not use player 4 .

In contrast, in a battle-by-battle player choice game, in race 3 , if team $B$ still has the dominant player 1 , team $A$ will certainly use player 4 if it still has her. If so, does team $B$ keep player 1 until race 3 ? Since the situation is similar to matching pennies, randomization is needed, so player 1 may be kept. Consider the following case in race 2 . Team $A$ won the 
first round, and still has players 2,3 , and 4 , while team $B$ has players 1 and 2 . Team $B$ must win the next two races to win the team contest.

\begin{tabular}{|c|c|c|c|}
\hline second race & & $\frac{3}{4}$ & $\frac{1}{4}$ \\
\hline & $\ell=1$ & 1 & 2 \\
\hline$\frac{3}{4}$ & 2,3 & 0.5 & 0.75 \\
\hline$\frac{1}{4}$ & 4 & 0.75 & 0 \\
\hline
\end{tabular}

Thus, with one additional player, our equivalence result no longer holds.

\subsection{Supermajority}

We assumed that the team that wins the majority of the battles is the winner of the game. One may wonder whether or not our results extend even if the winning team needs to win a supermajority (if no team wins a supermajority, then it is a draw). The following example reveals non-uniqueness of Nash equilibrium payoffs and the total randomization equilibrium is a Pareto-dominated equilibrium.

Example 4. Suppose that there are two battles and teams $A$ and $B$ have two players each, and the team that wins both battles is the winner. We assume the following exogenous probability matrix: ${ }^{7}$

$$
Q=\left(\begin{array}{ll}
q_{11} & q_{12} \\
q_{21} & q_{22}
\end{array}\right)=\left(\begin{array}{ll}
0.5 & 0.3 \\
0.7 & 0.5
\end{array}\right)
$$

Since there are only two orderings available for each player and a team wins only when both

\footnotetext{
'If players' efforts are endogeneized, we need to specify players' objective functions. In order to get the invariant winning probabilities of battles in that case, we need to assume that players care about winning the majority.
} 
players win, the payoff matrix is:

\begin{tabular}{|c|c|c|}
\hline & 12 & 21 \\
\hline 12 & $q_{11} q_{22},\left(1-q_{11}\right)\left(1-q_{22}\right)$ & $q_{12} q_{21},\left(1-q_{12}\right)\left(1-q_{21}\right)$ \\
\hline 21 & $q_{21} q_{12},\left(1-q_{21}\right)\left(1-q_{12}\right)$ & $q_{22} q_{11},\left(1-q_{22}\right)\left(1-q_{11}\right)$ \\
\hline
\end{tabular}$=$\begin{tabular}{|c|c|c|}
\hline & 12 & 21 \\
\hline 12 & $0.25,0.25$ & $0.21,0.21$ \\
\hline 21 & $0.21,0.21$ & $0.25,0.25$ \\
\hline
\end{tabular}

Thus, in this case, there are two Nash equilibria in pure strategies, $(12,12)$ and $(21,21)$, although there is also a total randomization mixed strategy equilibrium. With supermajority, this game is a coordination game with three Nash equilibria: it is no longer a zero-sum game. Thus, even Hamilton-Romano's uniquness result (Proposition 1) does not hold in this case. $\square$

However, our results hold for the cases of unequal score rule without a draw (for example, for team $A$ to win, it needs two-thirds of the battles, while team $B$ needs only one-third), then all equivalence results are preserved since the game is still a zero-sum game.

\subsection{Total Payoff Maximizing Team Leaders}

In the final example, we consider the case where team leaders maximize the team members' total payoffs instead of their teams' winning probabilities. We assume that players care about their team's winning probability. In this case, each member's effort cost needs to be taken into consideration explicitly. For this purpose, let the marginal costs of players $i$ and $j$ be $c_{i}$ and $c_{j}$, respectively, and let each battle's contest success function be a simple Tullock form: team $A$ 's winning probability in a battle played by $i$ and $j$ is specified as $q_{i j}=\frac{x_{i}}{x_{i}+x_{j}}$. When every player gets $V=1$ if her team wins, the equilibrium of a battle between $i$ and $j$ is described as $q_{i j}=\frac{c_{j}}{c_{i}+c_{j}}$ and $x_{i}=\frac{c_{j}}{\left(c_{i}+c_{j}\right)^{2}}$. We demonstrate by the following example that the total payoff maximizing team leaders may not randomize players totally in a battle-by-battle player choice game.

Example 5. Teams $A$ and $B$ have three players each, and their marginal costs are 2 except 
for one star player in team $A$ : her marginal cost is 1 . Consider the case where two mediocre players were matched in battle 1 and the team $A$ player won. Now, two team leaders are choosing which players play in the second battle. Essentially, team $A$ 's leader only has one choice: use the star player in the second battle or not. Team $A$ needs to win only one more game, so even if it loses in the second battle, it can still win with the winning probability of the third battle. Let $i_{2}$ and $j_{2}$ be the second battle players, and $i_{3}$ and $j_{3}$ be the third battle players. Then, the second battle's stake is $1-q_{i_{3} j_{3}}$, and $x_{i_{2}}=\left(1-q_{i_{3} j_{3}}\right) \frac{c_{j_{2}}}{\left(c_{i_{2}}+c_{j_{2}}\right)^{2}}$. Thus, team $A$ 's leader maximizes the following expected total payoff in this subgame.

$$
\begin{aligned}
W^{A 2} & =3\left(q_{i_{2} j_{2}}+\left(1-q_{i_{2} j_{2}}\right) q_{i_{3} j_{3}}\right)-x_{i_{2}}-\left(1-q_{i_{2} j_{2}}\right) x_{i_{3}} \\
& =3\left(\frac{c_{j_{2}}}{c_{i_{2}}+c_{j_{2}}}+\frac{c_{i_{2}}}{c_{i_{2}}+c_{j_{2}}} \frac{c_{j_{3}}}{c_{i_{3}}+c_{j_{3}}}\right)-\frac{c_{i_{3}}}{c_{i_{3}}+c_{j_{3}}} \frac{c_{j_{2}}}{\left(c_{i_{2}}+c_{j_{2}}\right)^{2}}-\frac{c_{i_{2}}}{c_{i_{2}}+c_{j_{2}}} \frac{c_{j_{3}}}{\left(c_{i_{3}}+c_{j_{3}}\right)^{2}}
\end{aligned}
$$

Thus, the expected total payoff by setting $c_{i_{2}}=1$ is

$$
W_{c_{i_{2}}=1}^{A 2}=3\left(\frac{2}{3}+\frac{1}{3} \times \frac{1}{2}\right)-\frac{1}{2} \times \frac{2}{9}-\frac{1}{3} \times \frac{1}{8}=2.3472
$$

while the one by setting $c_{i_{3}}=1$ is

$$
W_{c_{i_{3}}=1}^{A 2}=3\left(\frac{1}{2}+\frac{1}{2} \times \frac{2}{3}\right)-\frac{1}{3} \times \frac{1}{8}-\frac{2}{3} \times \frac{2}{9}=2.3102
$$

Thus, the total randomization is not an equilibrium in this subgame. This is because if the game ends early, the third player does not need to make any effort in a battle-by-battle player choice game. In contrast, in a one-shot ordering choice game, the total randomization is still a Nash equilibrium since all three games are played in a one-shot game. ${ }^{8} \square$

\footnotetext{
${ }^{8}$ When a team leader maximizes the total team payoff, the game is no longer a zero-sum game. So, there may be other Nash equilibria.
} 


\section{Conclusions}

In this paper, we show that $\mathrm{Fu}, \mathrm{Lu}$, and Pan's (2015) invariance results extend even if the team leaders strategically choose the order in which players are sent to the battlefield. The independence of each battle's winning probability extends as long as the zero homogeneity of the contest success function of each battle is satisfied. Additionally, somewhat surprisingly, the total randomization of player choice at any level is the equilibrium strategy irrespective of whether team leaders' choices are made as one-shot or battle-by-battle decisions. We also explore the robustness and limitations of our equivalence results by investigating several extensions: we found that considering ordering choice decisions add additional subtleties to the model.

\section{Appendix}

Here, we formally illustrate the way to show that Theorem 2 and Corollary 1 extend for any temporal structure of the player-choice game. Let a temporal structure be $\mathbf{n}=\left(n_{t}\right)_{t=1}^{\bar{t}}$ such that $\sum_{t=1}^{\bar{t}} n_{t}=2 n+1$, where $n_{t}$ is a positive integer: i.e., at each round $t=1, \ldots, \bar{t}, n_{t}$ battles take place simultaneously. This setup includes both one-shot ordering choice games $\left(\bar{t}=1\right.$ and $\left.n_{1}=2 n+1\right)$, and battle-by-battle player-choice games $\left(\bar{t}=2 n+1\right.$ and $n_{t}=1$ for all $t=1, \ldots, \bar{t})$. Now, we will consider a player-choice game in this temporal structure. Let $S_{t}$ be a collection of all corresponding states for round $t=1, \ldots, \bar{t}$. That is, a state $s_{t}=\left(k, \ell, h ; T^{A}, T^{B}\right) \in S_{t}$ holds if and only if $k=\left|T^{A}\right|=\left|T^{B}\right|=\sum_{t^{\prime}=t}^{\bar{t}} n_{t^{\prime}}$ and $\ell+h=k+1$. We use the functions $k\left(s_{t}\right)=k, \ell\left(s_{t}\right)=\ell, h\left(s_{t}\right)=h, T^{A}\left(s_{t}\right)=T^{A}$, and $T^{B}\left(s_{t}\right)=T^{B}$ to indicate the relevant information at state $s_{t}=\left(k, \ell, h ; T^{A}, T^{B}\right)$.

We start with the following definitions. Let $\Lambda_{t}\left(T^{\nu}\right) \equiv\left\{R_{t}^{\nu} \subseteq T^{\nu}:\left|R_{t}^{\nu}\right|=n_{t}\right\}$ be a collection of all possible subsets of $T^{\nu}$ with the cardinality of $n_{t}$. Each team leader $\nu$ 's action set at state $s_{t}$ is $\tilde{\Pi}^{\nu}\left(s_{t}\right)=\cup_{R_{t}^{\nu} \in \Lambda_{t}\left(T^{\nu}\left(s_{t}\right)\right)} \Pi\left(R_{t}^{\nu}\right)$, where $\Pi(R)$ is a collection of all permu- 
tations of set $R$. For $s_{t} \in S_{t}$, let $\triangle\left(\tilde{\Pi}\left(s_{t}\right)\right)$ be the sets of mixed strategies for leader $\nu=A, B$, and define $\sigma_{t}^{\nu}: S_{t} \rightarrow \triangle\left(\tilde{\Pi}^{\nu}\left(s_{t}\right)\right)$ such that $\sigma_{t}^{\nu}\left(s_{t}\right) \in\left(\tilde{\Pi}^{\nu}\left(s_{t}\right)\right)$ as the mixed strategy of the leader $\nu$ at state $s_{t}$. One possible subgame perfect equilibrium strategy is $\bar{\sigma}_{t}^{\nu}\left(s_{t}\right)=\frac{1}{\left|\tilde{\Pi}^{\nu}\left(s_{t}\right)\right|}(1,1, \ldots, 1) \in \triangle\left(\tilde{\Pi}^{\nu}\left(s_{t}\right)\right)$ for $\nu=A, B$, and all $t=1, \ldots, \bar{t}$. Finally, for any action $\pi_{t}^{\nu} \in \tilde{\Pi}^{\nu}\left(s_{t}\right)$, we denote $\check{R}\left(\pi_{t}^{\nu}\right) \subset T^{\nu}\left(s_{t}\right)$ as the set of players involved in the action $\pi_{t}^{\nu}$.

In order to generalize Theorem 2, we set an induction hypothesis: in each state $s_{t} \in$ $S_{t}$, (i) subgame perfect equilibria generate each possible matching of the leftover players $\mu_{t} \in M\left(T^{A}, T^{B}\right)$ occurs with the same probability, (ii) for all pair of players $(i, j) \in T^{A} \times$ $T^{B}$, equilibrium winning probability of $i$ is $\bar{q}_{i j}$, (iii) the total-randomization strategy profile $\left(\bar{\sigma}_{t^{\prime}}^{A}\left(s_{t}\right), \bar{\sigma}_{t^{\prime}}^{B}\left(s_{t}\right)\right)_{t^{\prime}=t}^{\bar{t}}$ is a subgame-perfect equilibrium; and (iv) team $A^{\prime}$ 's expected winning probability in the beginning of each state $s_{t} \in S_{t}$ is $\bar{P}^{A}\left(s_{t}\right)$.

For $t=\bar{t}$, the hypothesis holds. Supposing that for all $t^{\prime}=t+1, \ldots, \bar{t}$, the hypothesis also holds, we focus on round $t^{\prime}=t$.

In each state $s_{t} \in S_{t}$, we need to consider every possible combination of player choices in the next round. For each choice combination $\left(\pi_{t}^{A}, \pi_{t}^{B}\right)$ with $\check{R}\left(\pi_{t}^{A}\right) \subset T^{A}\left(s_{t}\right)$ and $\check{R}\left(\pi_{t}^{B}\right) \subset$ $T^{B}\left(s_{t}\right)$, depending on the winner of the battle, the next state $s_{t+1}$ will be in the set $S_{t+1}\left(s_{t} ; \check{R}\left(\pi_{t}^{A}\right), \check{R}\left(\pi_{t}^{B}\right)\right)=\left\{s_{t+1}=\left(k\left(s_{t}\right)-n_{t}, \ell^{\prime}, h^{\prime} ; T^{A}\left(s_{t}\right) \backslash \check{R}\left(\pi_{t}^{A}\right), T^{B}\left(s_{t}\right) \backslash \check{R}\left(\pi_{t}^{B}\right)\right)\right.$ with $\ell^{\prime}+$ $h^{\prime}=k\left(s_{t}\right)-n_{t}+1, \ell\left(s_{t}\right)-n_{t} \leq \ell^{\prime} \leq \ell\left(s_{t}\right)$, and $\left.h\left(s_{t}\right)-n_{t} \leq h^{\prime} \leq h\left(s_{t}\right)\right\}$. By the induction hypothesis, $\bar{P}\left(s_{t+1}\right)$ is team $A$ 's winning probability once state $s_{t+1}$ occurs. By this, given a choice combination $\left(\pi_{t}^{A}, \pi_{t}^{B}\right)$, we can calculate the probability for every pair $(i, j) \in R_{t}^{A} \times R_{t}^{B}$ to be pivotal. We can show that the probability of this battle being pivotal is the same for both players. Therefore, by Lemma 1 , $i$ 's equilibrium winning probability is $\bar{q}_{i j}$. Then, we can create a payoff matrix for round $t$ 's ordering choice game. If team $B$ totally randomizes the ordering of players, every $\mu_{t} \in M\left(\Lambda_{t}\left(T^{A}\right), \Lambda_{t}\left(T^{B}\right)\right)$ shows up with equal probabilities no matter which ordering team $A$ chooses. Similarly, let team $A$ also randomize its ordering totally. This total randomized strategy combination is subgame perfect. Again by induction, we can show that every $\mu_{t} \in M\left(\Lambda_{t}\left(T^{A}\right), \Lambda_{t}\left(T^{B}\right)\right)$ occurs with the same probability, and team 
$A$ 's expected winning probability in the beginning of each state $s_{t} \in S_{t}$ is $\bar{P}^{A}\left(s_{t}\right)$. To show this formally, first we define $\check{W}(k, \xi) \equiv\left\{S \in 2^{\{1, \ldots, k\}}|| S \mid=\xi\right\}$ and

$$
\check{P}\left(\mu_{t}, \xi\right)=\sum_{S \in \breve{W}\left(k_{t}, \xi\right)}\left(\prod_{r \in S} \bar{q}_{i_{r} \mu_{t}\left(i_{t}\right)} \prod_{r \notin S}\left(1-\bar{q}_{i_{r} \mu_{t}\left(i_{r}\right)}\right)\right),
$$

which is probability of winning $\xi$ out of $n_{t}$ battles given a matching $\mu_{t}$. By the induction assumptions, the expected payoff of choosing any $\pi_{t}^{A} \in \tilde{\Pi}^{\nu}\left(s_{t}\right)$ given team $B$ 's strategy is $\bar{\sigma}_{t}^{B}\left(s_{t}\right)$ is

$$
\begin{aligned}
& \frac{1}{\left|\tilde{\Pi}^{B}\left(s_{t}\right)\right|} \sum_{\pi_{t}^{B} \in \tilde{\Pi}^{B}\left(s_{t}\right)}\left(\sum_{\xi=0}^{n_{t}} \check{P}\left(\mu\left(\pi_{t}^{A}, \pi_{t}^{B}\right), \xi\right) \times\right. \\
& \left.\bar{P}\left(s_{t+1}\left(k_{t+1}, \ell\left(s_{t}\right)-\xi, h\left(s_{t}\right)-n_{t}+\xi\right), T^{A}\left(s_{t}\right) \backslash \check{R}\left(\pi_{t}^{A}\right), T^{B}\left(s_{t}\right) \backslash \check{R}\left(\pi_{t}^{B}\right)\right)\right) \\
= & \frac{1}{C_{n_{t}}^{\left|T^{B}\left(s_{t}\right)\right|} n_{t} !} \frac{1}{k\left(s_{t}+1\right) !} \sum_{\pi_{t}^{B} \in \tilde{\Pi}^{B}\left(s_{t}\right)}\left(\sum_{\xi=0}^{n_{t}} \check{P}\left(\mu\left(\pi_{t}^{A}, \pi_{t}^{B}\right), \xi\right) \times\right. \\
= & \frac{1}{\left|T^{B}\left(s_{t}\right)\right| !} \sum_{\pi_{t}^{B} \in \tilde{\Pi}^{B}\left(s_{t}\right)} \sum_{\mu \in M\left(T^{A}\left(s_{t}\right), T^{B}\left(s_{t}\right) ; \mu_{t}\right)} \tilde{P}\left(\mu ; k\left(s_{t}\right), \ell\left(s_{t}\right)\right) \\
= & \bar{P}\left(s_{t}\right),
\end{aligned}
$$

where $M\left(T^{A}\left(s_{t}\right), T^{B}\left(s_{t}\right) ; \mu_{t}\right)$ is the set of matching between $T^{A}\left(s_{t}\right)$ and $T^{B}\left(s_{t}\right)$ such that the first $n_{t}$ battles are the same as $\mu_{t}$. This completes the induction argument.

\section{References}

Barbieri, S., and M. Serena (2019): "Winners' Efforts in Team Contests," Working Paper. 
Fu, Q., and J. Lu (2018) "On Equilibrium Player Ordering in Dynamic Team Contests," Working Paper.

Fu, Q., J. Lu, and Y. Pan (2015): "Team Contests with Multiple Pairwise Battles," The American Economic Review, 105(7), 2120-2140.

Häfner, S. (2017): "A Tug-of-War Team Contest," Games and Economic Behavior, 104, 372-391.

Hamilton, J., and R. E. Romano (1998): "Equilibrium Assignment in Pairwise Team Contests: How to Form Political Slates and Tennis Teams," European Journal of Political Economy, 14(1), 101-114.

Harris, C., and J. Vickers (1987): "Racing with Uncertainty," Review of Economic Studies, $54(1), 1-21$.

Klumpp, T., and M. Polborn (2006): "Primaries and the New Hampshire Effect," Journal of Public Economics, 90(6-7), 1073-1114.

Klumpp, T., K. Konrad, and A. Solomon (2019): "The Dynamics of Majoritarian Blotto Games," Game and Economic Behavior, 117, 402-419.

Konrad, K. A., and D. Kovenock (2009): "Multi-Battle Contests," Game and Economic Behavior, 66(1), 256-274.

Konrad, K. A. (2018): "Budget and Effort Choice in Sequential Colonel Blotto Campaigns," CESifo Economic Studies, 64(4), 555-576.

Kovenock, D., and B. Roberson (2012) "Conflicts with Multiple Battlefields," in M. R. Garfinkel and S. Skaperdas (Eds.), Oxford Handbook of the Economics of Peace and Conflict, Oxford University Press, New York, 503-531. 
Malueg, D., and A. Yates (2005) "Equilibria and comparative statics in two-player contests," European Journal of Political Economy 21(3), 738-752. 